\title{
Vorwort des Editor-in-Chief
}

\author{
Gerhard Mayer \\ Leoben, Österreich
}

Online publiziert am 4. April 2016

Ergänzend zu den Beiträgen des Heftes 02/2016 konnten wir für das vorliegende Heft 04/2016 weitere Beiträge aus der Reihe der Vorträge des "15. Altbergbaukolloquiums" gewinnen, das von 5. bis 7. November 2015 in Leoben abgehalten wurde.

Besonders hervorheben möchte ich in diesem Zusammenhang den Beitrag von LeopoldWeber, Wolfgang Lenhardt, Gregor Götzl, Julia Weilbold, Heinz Reitner und Franz Möstl, in dem intelligente und multifunktionelle Nachnutzung des Altbergbaustandortes Arzberg dargestellt wird.

Die beiden Beiträge von Michael Heitfeld, Frank Denys, Johannes Klünker, Peter Rosner, Jaap Spaans zum Thema "Systematische Erfassung und Bewertung von Spätfolgen des Steinkohlenbergbaus in Südlimburg/Niederlande" und von Andreas Koschare, Katharina Telenga, Jutta Schliebs, Jörg Mittrach zum Thema "Ganzheitliche Betrachtung bei Analyse, Planung und Ausführung von Sanierungen - Risikomanagement im Altbergbau“ zeigen die länderübergreifende und ganzheitliche Betrachtungsweise der Altbergbauthematik besonders anschaulich.

Der Autor Nikolaus August Sifferlinger vom Lehrstuhl für Bergbaukunde, Bergtechnik und Bergwirtschaft der Montanuniversität befasst sich in seinem Beitrag „Proximity detection - Schutzeinrichtung für Gefahrenzonen im Untertageberg- und Tunnelbau" mit einer sicherheitlichen Thematik. Dieser Bereich wurde an der Montanuniversität auf breiter Basis in Forschung und Lehre etabliert.

Abgerundet wird die Folge der Beiträge des vorliegenden Heftes durch Arbeit vonTobias Braun, Alexander Hennig zum Thema "Untersuchungen zur Betriebsstruktur deutscher Natursteintagebaue." Beide Autoren sind am Institute of Mineral Resources Engineering der RWTH Aachen tätig.

Mit freundlichem Glückauf!

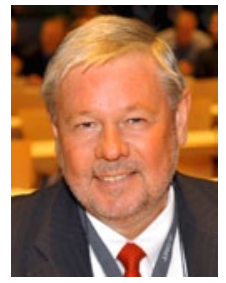

Gerhard Mayer 